\title{
Severe clinical outcome is uncommon in Clostridium difficile infection in children: a retrospective cohort study
}

\author{
Kevin L Schwartz ${ }^{1 *}$, Ilyse Darwish², Susan E Richardson ${ }^{3}$, Michael R Mulvey ${ }^{4}$ and Nisha Thampi ${ }^{1,5}$
}

\begin{abstract}
Background: Clostridium difficile infection (CDI) is the most common cause of health care-associated diarrhea in children and adults. Although serious complications of CDI have been reported to be increasing in adults, this trend has not yet been demonstrated in children. The purpose of this study was to examine the features of CDI in a pediatric population, with special attention to the occurrence of CDI-related severe outcomes.

Methods: A chart review was conducted for patients with C. difficile infection detected by cytotoxin assay between August, 2008 and July, 2012. Basic demographics, mode of acquisition (nosocomial versus community), laboratory and clinical features, treatment, and outcome data were collected. Pulsed-field gel electrophoresis and polymerase chain reaction detection of toxin $A(t c d A)$, toxin $B(t c d B)$, binary toxin $(c d t B)$ and $t c d C$ genes were performed on isolates from nosocomial cases by the National Microbiology Laboratory, Winnipeg, Manitoba.

Results: Ninety percent of children with CDI experienced resolution of symptoms by 30 days after disease onset and $2 \%$ experienced a severe outcome. There were no cases where colectomy was performed for CDI, and only one case where CDI contributed to death. Various combinations of clinical and laboratory features were not predictive of a severe outcome. Seventy-four percent of cases were nosocomial-associated. Among all cultured strains, the NAP4 strain occurred most frequently (24\%), followed by NAP1 (11\%). There was no association between strain type and clinical outcome; however, relapses were significantly more frequent in NAP4-infected children.

Conclusions: Severe outcomes due to CDI are uncommon in children compared to adults. Further prospective pediatric studies on CDI in community and hospital settings are required to better understand risk factors, optimal treatment and the significance of NAP4 in pediatric CDI.
\end{abstract}

Keywords: Clostridium difficile, North American pulsed-field type, Pediatrics, Child

\section{Background}

Clostridium difficile is the most common cause of diarrhea in hospitalized children [1,2], usually associated with antibiotic use. Over the last decade incidence rates of nosocomial C. difficile infection (CDI) in adults have increased in most geographical locations [3]. Accompanying the rise in incidence has been an increase in the rate of colectomies performed for $\mathrm{CDI}$, length of stay (LOS), hospital costs, and attributable mortality [4-6] associated with $C$. difficile infection. This rise in severity

\footnotetext{
* Correspondence: Kevin.schwartz@sickkids.ca

'Division of Infectious Diseases, The Hospital for Sick Children, University of Toronto, 555 University Ave, Rm 7306, Toronto, ON M5G 1X8, Canada Full list of author information is available at the end of the article
}

has been largely linked to the emergence of the North American pulse-field gel electrophoresis type 1, restriction endonuclease analysis group $\mathrm{BI}$, polymerase chain reaction (PCR) ribotype 027 (NAP1/BI/027) strain [5,7].

The epidemiology of pediatric $C$. difficile has been less well characterized, however recent data suggest a rise in the incidence rate of nosocomial CDI, from 4.4 to 6.5 cases per 10,000 patient-days in the United States (US) between 2001 and 2006 [8]. Other authors have documented a rise in incidence of 9\% [9] to 14\% [10] per year. A similar rise in community-associated cases has also been observed [11,12]. One U.S. pediatric study showed an increase from 2.2 to 23.5 cases of CDI per 100,000 persons over the previous two decades [12]. 
Despite the more frequent diagnosis of CDI, there has not been an increase in disease severity as measured by LOS, colectomy rate, and mortality rate $[9,10,13]$. However, longer LOS and higher colectomy rates occur in children with CDI compared to non-CDI hospitalized controls matched for demographics and comorbid conditions [9].

Given our clinical observation that CDI in children is rarely associated with a severe clinical outcome, as compared to adults, we set out to characterize severe outcomes and CDI-associated complications at a pediatric hospital providing primary (self-referred cases) to tertiary (regional referral center) care to a large population base.

\section{Methods}

A retrospective chart review was conducted at a 370-bed pediatric academic hospital in Toronto, Canada. Our institution services a varied pediatric population, ranging from the well child to the immune compromised child. This hospital performs 110 autologous and allogeneic hematopoietic stem cell transplants annually, in addition to solid organ transplants (liver, kidney, heart, lung, and small bowel). Patients from 1 through 17 years of age with a positive $C$. difficile cytotoxin assay in the stool between August 1, 2008 and July 31, 2012, were identified from microbiology records. Our laboratory restricts testing to children greater than one year of age.

Charts from the first presentation of CDI were reviewed and information related to basic demographics, laboratory and clinical features, $C$. difficile-directed antibiotic use, and clinical outcome were extracted. Clinical and laboratory features included stool frequency and consistency. Clinically-based definitions for diarrhea were modified from the Canadian Nosocomial Infection Surveillance Program (CNISP) definitions [14] for use in children, and were defined as: severe: 6 or more watery stools in a 36-hour period; moderate: greater than 2 loose stools, but not meeting the definition for severe; mild: a change in stooling pattern to 1-2 loose stools daily; no diarrhea or not documented. Risk factors for severity of infection were defined as: presence of blood in stool, fever $\geq 38^{\circ} \mathrm{C}$, leukocytosis $\geq 15 \times 10^{9} / \mathrm{L}$, hypoalbuminemia $\leq 25 \mathrm{~g} / \mathrm{L}$, and creatinine above the normal limit according to age [15]. A severe outcome was defined as the need for intensive care unit (ICU) admission, colectomy, or death within 30 days of a positive $C$. difficile cytotoxin assay [14]. Each severe outcome was evaluated and designated as unrelated to, contributing to, or a direct effect of CDI by authors, KS and ID, and confirmed by two other investigators, SR and NT. Other clinical outcomes assessed at 30 days after disease onset included date of discharge from hospital, presence or absence of symptoms, or ongoing treatment directed at $C$. difficile. Relapse was defined as recurrence of previously resolved symptoms with a positive test for $C$. difficile, two or more weeks from the onset of the initial episode, whether or not the patient had been treated with antibiotics. If patients required re-admission for relapse, their charts were reviewed to capture any severe outcome, however, each patient was only included in the study once. C. difficile was categorized as community-associated if symptoms developed within 72 hours of admission, with no hospitalization in the previous 8 weeks, consistent with CNISP [14]. This study was approved by the Quality Improvement Review Board at the Hospital for Sick Children, Toronto, Canada. Individual patient/guardian consent was not obtained.

Statistically significant differences between groups were assessed by the chi-squared test or Fisher's exact test, where appropriate. A p-value $<0.05$ was considered statistically significant. The incidence of nosocomialassociated disease was calculated to be the number of cases of CDI per 10,000 hospital inpatient days.

\section{Laboratory methods}

C. difficile toxin was detected using a Vero cell cytotoxicity assay as previously described [16]. Stools from nosocomial CDI cases were frozen at $-80^{\circ} \mathrm{C}$, and referred to the National Microbiology Laboratory, Winnipeg, Canada, for culture, typing and further analysis as part of a larger study through CNISP [14]. Isolates were typed using pulsed-field gel electrophoresis (PFGE) to determine North American pulsed-field (NAP) types, and PCR to detect the presence of genes for toxin $A$ and $\mathrm{B}$ ( $t c d A$ and $t c d B$, respectively), binary toxin $(c d t B)$, and size alterations of the toxin gene regulator $(t c d C)$ [7]. Minimum inhibitory concentrations of each strain to a panel of antibiotics (metronidazole, clindamycin, vancomycin, rifampin, moxifloxacin, tigecycline) were determined by the Etest method [17].

\section{Results}

From August 1, 2008 to July 31, 2012 there were 5751 stool samples tested for $C$. difficile toxin by cytotoxin assay, with 435 positives and 299 individual patients analyzed. During the study period, the nosocomial CDI rate was 8.3 per 10,000 patient days. This trend did not change in any age group or year over the 4-year study period. Hospital-associated infections accounted for 222 (74\%) of cases, and 77 (26\%) were community-associated.

Demographic, clinical and laboratory findings are summarized in Table 1. Males accounted for $58 \%$ of the total number of cases and the median age was 6.5 years (interquartile range, 3-13 years). Nearly $40 \%$ of cases had an underlying malignancy, a third of which had undergone hematopoietic stem cell transplant (HSCT). Inflammatory bowel disease (IBD) comprised $10 \%$ of 
Table 1 Characteristics of 299 children with Clostridium difficile cytotoxin detected in stool

\begin{tabular}{|c|c|}
\hline Category & n (\%) \\
\hline \multicolumn{2}{|l|}{ Demographic Characteristics } \\
\hline Age (years) & $6.5(3-13)^{\dagger}$ \\
\hline $1-5$ & $125(42)$ \\
\hline $6-11$ & $83(28)$ \\
\hline $12-17$ & $91(30)$ \\
\hline Male & $173(58)$ \\
\hline Underlying medical condition & $206(69)$ \\
\hline Malignancy & $78(26)$ \\
\hline Hematopoietic stem cell transplant & $40(13)$ \\
\hline Chromosomal/genetic syndrome & $33(11)$ \\
\hline Inflammatory bowel disease & $31(10)$ \\
\hline Solid organ transplant & $24(8)$ \\
\hline Cystic fibrosis & $8(3)$ \\
\hline Other* & $8(3)$ \\
\hline Congenital heart disease & $6(2)$ \\
\hline Hirschsprung's disease & $2(1)$ \\
\hline Hospital-associated CDI & $222(74)$ \\
\hline \multicolumn{2}{|l|}{ Clinical features } \\
\hline Fever $\geq 38.0^{\circ} \mathrm{C}$ & $140(47)$ \\
\hline Abdominal pain & $106(35)$ \\
\hline Bloody stool & $51(17)$ \\
\hline Ileus & $7(2)$ \\
\hline \multicolumn{2}{|l|}{ Diarrhea } \\
\hline Severe & $81(27)$ \\
\hline Moderate & $132(44)$ \\
\hline Mild & $65(22)$ \\
\hline None/no documentation & $21(7)$ \\
\hline \multicolumn{2}{|l|}{ Laboratory features } \\
\hline White blood cells $\geq 15 \times 10^{9} / \mathrm{L}$ & $64(21)$ \\
\hline Creatinine > normal level (adjusted for age) & $40(13)$ \\
\hline Albumin $\leq 25 \mathrm{~g} / \mathrm{L}$ & $45(15)$ \\
\hline CDI-directed antimicrobial therapy & $240(80)$ \\
\hline
\end{tabular}

${ }^{\dagger}$ Median (interquartile range).

*Other underlying medical conditions: Aplastic anemia (4), Juvenile idiopathic arthritis (3), and biliary atresia (1).

cases. Among children with IBD, 55\% (17/31) had community-associated disease.

Mild, moderate, or severe diarrhea was seen in $22 \%$, $44 \%$, and $27 \%$ of children with CDI, respectively (Table 1). Clinical severity was similar in communityand nosocomial-associated cases. Two or more risk factors for severe disease were noted among 89 children (30\%). However, these cases were not significantly associated with severe outcomes, compared to those who had less than two risk factors (Table 2). The relapse rate was $17 \%$ overall. Eighty per cent of children with $C D$ toxin in their stool received antibiotic therapy directed at CDI, usually metronidazole.

At thirty days following the onset of symptoms, $89 \%$ of patients were discharged or had resolution of symptoms, $2 \%$ had a severe outcome (5 ICU admissions, 1 CDI-associated death), and $9 \%$ had ongoing diarrhea or were still receiving treatment, more than half of whom had underlying IBD or malignancy (Table 2). The 5 children admitted to the ICU required management of ileus, upper gastrointestinal bleeding, or septic shock. Colectomy was not performed for any reason on any patient in the cohort during the study period.

There was one death during the study period, to which CDI was deemed to contribute. This was in a 7-year-old child following HSCT who developed severe diarrhea followed by septic shock and ileus. He was admitted to the ICU, grew Enterobacter cloacae from his blood with C. difficile toxin identified in the stool on the same day. The child was unstable and no surgical intervention was considered. Within 48 hours of admission to ICU the child died from severe sepsis. The primary cause of death was felt to be gram-negative sepsis with CDI contributing to death.

PFGE data were available for 90 cultured C. difficile isolates from 129 nosocomial cases submitted to CNISP between August 1, 2008 and July 31, 2012 (Table 3). Thirty-four isolates (38\%) did not have a fingerprint pattern that fell into a NAP cluster designation. NAP4 was the most common strain type $(n=22,24 \%)$, followed by NAP1 $(n=10,11 \%)$. Relapse rates were $41 \%$ and $15 \%$ for NAP4 and non-NAP4 strains, respectively, which was statistically significant $(p=0.009)$. There were no significant differences in symptom severity, the need for ICU admission, surgical consultation, or death based on strain type. Of the 90 isolates, all had toxin A and B detected by PCR. Binary toxin was detected in $16(18 \%)$ of 90 isolates, and in 9 out of 10 of the NAP1 strains. The $t c d C$ gene was detected in all isolates; all NAP1 strains had the characteristic 18 bp deletion. All isolates were susceptible to metronidazole, vancomycin, rifampin, and tigecycline. In addition, $60 \%$ of isolates were intermediate or resistant to clindamycin, and $9 \%$ were intermediate or resistant to moxifloxacin. Of the NAP1 and NAP4 strains, $30 \%$ and $9 \%$ were intermediate or resistant to moxifloxacin, respectively (Table 3 ).

\section{Discussion}

In this study, we describe a cohort of 299 hospitalized children, 1-17 years of age, with $C$. difficile infection at a large tertiary care centre. Three quarters of the cases were nosocomial in origin and occurred among children with chronic underlying medical conditions, many of whom had compromised immune systems or inflammatory 
Table 2 Outcomes of 299 children with C. difficile infection

\begin{tabular}{|c|c|c|c|c|c|}
\hline Outcome & Total, n (\%) & $\begin{array}{l}2 \text { or more risk } \\
\text { factors }^{\dagger}, \mathrm{n}(\%)\end{array}$ & $\begin{array}{l}\mathrm{CDI} \text { treatment } \\
\text { received, } \mathrm{n}(\%)\end{array}$ & $\begin{array}{l}\text { Underlying diagnosis } \\
\text { of IBD, } n(\%)\end{array}$ & $\begin{array}{l}\text { Underlying diagnosis } \\
\text { of cancer/following } \\
\text { BMT, } \mathbf{n}(\%)\end{array}$ \\
\hline Discharged or resolution of symptoms & $269(90)$ & $77(29)$ & $211(71)$ & $22(8)$ & $107(40)$ \\
\hline Ongoing diarrhea and/or treatment & $28(9)$ & $12(43)$ & $27(96)$ & $9(32)^{*}$ & $7(25)$ \\
\hline ICU admission & $5(2)$ & $3(60)$ & $5(100)$ & $0(0)$ & $4(80)$ \\
\hline Death $^{\neq}$ & $1(0.3)$ & $1(100)$ & $1(100)$ & $0(0)$ & $1(100)$ \\
\hline Relapse & $52(17)$ & $13(25)$ & $43(83)$ & $5(10)$ & $24(21)$ \\
\hline
\end{tabular}

*Significant at $\mathrm{p}<0.05$. Children with IBD and CDI were more likely to have ongoing diarrhea compared to children without IBD by Chi-squared test. No other significant differences were identified.

${ }^{\dagger}$ Risk factors include fever $>38^{\circ} \mathrm{C}$, bloody stool, hypoalbuminemia $\leq 25 \mathrm{~g} / \mathrm{L}$, leukocytosis $\geq 15 \times 10^{9} / \mathrm{L}$, and renal dysfunction defined as creatinine above normal limits for age. There were no significant differences in outcomes $(p>0.05)$ in children with and without 2 or more risk factors.

${ }^{\ddagger}$ Death to which CDI contributed.

$\mathrm{CDI}=C$. difficile infection; ICU = intensive care unit; $\mathrm{IBD}=$ inflammatory bowel disease; $\mathrm{BMT}$ = bone marrow transplant.

bowel disease. We found that severe clinical outcomes related to CDI occurred in only $2 \%$ of our cases, despite the presence of serious underlying illness in $50 \%$ of cases. Colectomy was not performed in any child, few were admitted to the ICU, and CDI was felt to contribute to only one death in the cohort. Most children fully recovered from their illness by 30 days after disease onset, irrespective of whether the illness was nosocomial or communityassociated, the severity of illness, or treatment received. Of the 9\% with ongoing diarrhea, more than half had underlying IBD or malignancy, which may have confounded the determination of etiology of persistent symptoms. We also observed a high prevalence of NAP4 among typeable isolates in our pediatric cohort, which is in contrast to the high prevalence of NAP1 strains in adults from similar geographic areas [18].

The incidence rate of nosocomial CDI (8.3 cases per 10,000 patient days) did not change significantly over the study period. This is in contrast to studies of American children, in which a 9\% yearly rise in incidence of CDI has been reported $[8,19]$.

The severity and outcome of CDI in children is poorly understood compared to adults. Pediatric data comparing children with CDI to matched controls have shown longer LOS, higher hospital costs, and higher rates of colectomy $[8,9]$. Administrative data sets including thousands of children with CDI have indicated colectomy rates ranging from 0.35 to $1.2 \%$ [8,9,20]. Adjusted for comorbidities, children with CDI in a large database study had a 1.36 fold increased risk of undergoing colectomy compared to controls without CDI [9]. One of the challenges with such administrative database studies is ascertaining the reasons for colectomy. It is possible that a number of the colectomies were performed for underlying IBD or for another clinical indication. If $C$. difficile is detected during an IBD exacerbation, it has been shown to be a marker for severity and increased inhospital mortality [21]. However, among both pediatric patients with IBD and non-IBD controls, toxigenic $C$. difficile is frequently detected irrespective of symptoms [22]. As a result, some of the complications seen in pediatric CDI are possibly accounted for by underlying comorbidities.

In our study, a small number of children (2\%) suffered a severe outcome. This is in marked contrast to adult CDI, where severe outcomes have been reported in 8-20\% $[7,23]$ and $C$. difficile-attributed or associated mortality has been reported in approximately $5-9 \%$ of cases, especially among those harboring the NAP1 strain $[4,7,24,25]$.

In contrast to reports of adult CDI, NAP4 was the most common strain type in our pediatric cohort, accounting for $24 \%$ of nosocomial isolates. NAP1 was the second most common at $11 \%$. Similar rates of NAP1 have recently been reported among children in the US

Table 3 North American Pulsed-field types in 90 children with nosocomial C. difficile infection and organism available for testing

\begin{tabular}{lcccc}
\hline Strain & Total, $\mathbf{n}(\%)$ & Relapse, $\mathbf{n}(\%)$ & Severe outcome $^{\dagger}, \mathbf{n}(\%)$ & Moxifloxacin susceptible, $\mathbf{n}(\%)$ \\
\hline NAP4 & $22(24)$ & $9(41)^{*}$ & $0(0)$ & $20(91)$ \\
NAP1 & $10(11)$ & $1(10)$ & $1(10)$ & $7(70)$ \\
Other NAP type ${ }^{\ddagger}$ & $24(27)$ & $2(8)$ & $0(0)$ & $22(92)$ \\
No NAP type & $34(38)$ & $7(21)$ & $2(6)$ & $33(97)$ \\
Total & $\mathbf{9 0}$ & $\mathbf{1 9 ( 2 1 )}$ & $\mathbf{3 ( 3 )}$ & $\mathbf{8 2 ( 9 1 )}$ \\
\hline
\end{tabular}

*p $=0.009$.

${ }^{+}$Severe outcome $=\mathrm{ICU}$ transfer, colectomy, or death within 30 days of a positive $C$. difficile cytotoxin assay.

${ }^{\ddagger}$ Other NAP $=\operatorname{NAP} 10(n=4), \operatorname{NAP} 11(n=8), \operatorname{NAP6}(n=3), \operatorname{NAP7}(n=2), \operatorname{NAP} 2(n=3), \operatorname{NAP} 8(n=2), \operatorname{NAP} 12(n=2)$. 
[26,27]. The trend across Canada in recent years has been toward an increasing incidence of NAP1 among adults with CDI, from $28.5 \%$ (2005) to $43.5 \%$ (2010), with the highest prevalence of $51 \%$ in eastern Canada [18].

Neither NAP4 nor NAP1 was associated with more severe illness in our cohort, although relapse rate was significantly higher in patients with NAP4 compared to non-NAP4 strains. However, we could not determine whether the relapses were caused by the same strain. In contrast, a large Canadian study of adult CDI showed that severe outcomes occurred significantly more frequently in patients between the ages of 60 and 90 years of age who were infected with the NAP1 strain, compared to other strains [7]. However, the importance of the NAP1 strain in severity of disease has been challenged by case-control studies that were unable to demonstrate any difference in disease severity with NAP1 infection compared to other strains [23]. From our data we were unable to associate the high prevalence of NAP4 with a cluster of cases, limited in time or geography. As this is the first report of NAP4 predominance in pediatric CDI, further investigation will be necessary to elucidate the role of NAP4 in disease in children and to better determine associations with disease relapse.

Another important difference between children and adults in Canada is the rate of moxifloxacin susceptibility. In our cohort $91 \%$ of all strains (91\% of NAP4 and $70 \%$ of NAP1 strains) were susceptible to moxifloxacin. In contrast, only $33 \%$ and $17 \%$ of all strains, and NAP1 strains, respectively, were susceptible to moxifloxacin in a predominantly adult Canadian study [14]. It should also be noted that the PFGE subtype of the NAP1 isolates identified in this study were not the common NAP1 PFGE subtype observed across Canada which is typically moxifloxacin-resistant. The lower use of fluoroquinolones in pediatrics could be driving this observation.

There are no established pediatric predictors for disease severity. Our study demonstrated that proven adult risk factors of fever, bloody stools, leukocytosis, renal dysfunction, and hypoalbuminemia were common in our population. These so-called risk factors were not associated with disease severity or complications and were likely related to the underlying conditions of malignancy and IBD. This finding has been identified by other authors who have proposed new criteria for pediatric CDI, which require further study and validation [28].

Most infections were associated with current or previous hospitalization, and occurred predominantly among children with underlying immunocompromising conditions. Community-associated infection was observed in $26 \%$ of children, more than half of whom had IBD. These findings represent our population of children with high rates of comorbid conditions. Communityassociated disease is harder to assess in this setting and would require a community-based study to determine incidence. A recent population-based study in the U.S. identified that $75 \%$ of pediatric CDI was communityassociated, suggesting that hospital-based incidence rates likely underestimate true CDI rates in children [12]. However, in our study, we were unlikely to miss complicated cases, as we expect most of these children to have been referred to our centre from the community. Given the lack of data and the increasing incidence of communityassociated disease [11,12], future pediatric CDI research should focus on this issue.

Our study has several limitations and biases inherent to retrospective reviews. Findings were limited to information available from the charts. Almost 30\% of children in this study would not have met adult criteria for disease by having less than 3 loose stools per day. However as there are no well-defined pediatric criteria for significant disease and, as the goal of this study was to detect any CDI related complication, we included all children with $C$. difficile detected in stools sent for clinical reasons. The cytotoxic assay is very specific and correlates well with disease but lacks sensitivity compared to PCR or to cytotoxic culture (toxin assay on culture positive $C$. difficile). Therefore it is possible some cases were missed. Sixty percent of nosocomial isolates were sent for reference typing, but in only half of these $C$. difficile was recovered on culture, resulting in a complete clinical with strain-typing dataset of $30 \%$ of the included children in the study. Antibiotic exposure data was missing for too many patients from which to draw meaningful conclusions regarding specific risk factors, particularly in community-associated disease. This will be important data to collect in future prospective studies on CDI in children.

Our study has a number of strengths. We were able to show the relationship of pediatric CDI with generally benign clinical outcomes. Furthermore since our hospital excludes testing in infants less than 1 year of age and our laboratory was utilizing a cytotoxin assay during the study period, we have confidence that these cases had a high probability of representing true infection as opposed to colonization.

\section{Conclusions}

This study describes a population of 299 children with nosocomial and community-associated CDI over a fouryear period. Children had a much lower rate of severe complications of CDI than that reported in adults. This is the first report to describe NAP4 as the predominant nosocomial strain type in a pediatric institution, and its association with relapse.

Competing interest

The authors declare that they have no competing interests. 


\section{Authors' contributions}

KS drafted the manuscript, assisted in collecting and analyzing data. ID collected and analyzed data as well as assisted in editing. SR assisted in design of the study, coordination, and helped draft and review the manuscript. MM performed the sequencing, PCR, and sensitivity analysis. NT assisted in design of the study, data collection, and helped draft and review the manuscript. All authors contributed significantly to editing the manuscript and approved it in its current form.

\section{Acknowledgments}

We would like to thank Tim Du and Romeo Hizon for their diligent work in the National Microbiology Laboratory on the C. difficile isolates as well as Rick Wray, Anne Matlow, and the Infection Prevention and Control Team at the Hospital for Sick Children.

\section{Author details}

'Division of Infectious Diseases, The Hospital for Sick Children, University of Toronto, 555 University Ave, Rm 7306, Toronto, ON M5G 1X8, Canada. ${ }^{2}$ Sandra A. Rotman Laboratories, University Health Network, University of Toronto, Toronto, ON, Canada. ${ }^{3}$ Division of Microbiology, Department of Paediatric Laboratory Medicine, The Hospital for Sick Children, University of Toronto, Toronto, ON, Canada. ${ }^{4}$ National Microbiology Laboratory, Winnipeg, MB, Canada. ${ }^{5}$ Division of Infectious Diseases, Mount Sinai Hospital, University of Toronto, Toronto, Canada.

Received: 12 September 2013 Accepted: 24 January 2014 Published: 31 January 2014

\section{References}

1. Langley JM, LeBlanc JC, Hanakowski M, Goloubeva O: The role of Clostridium difficile and viruses as causes of nosocomial diarrhea in children. Infect Control Hosp Epidemiol 2002, 23(11):660-664.

2. Klein EJ, Boster DR, Stapp JR, Wells JG, Qin X, Clausen CR, et al: Diarrhea etiology in a Children's Hospital Emergency Department: a prospective cohort study. Clin Infect Dis 2006, 43(7):807-813.

3. Cohen SH, Gerding DN, Johnson S, Kelly CP, Loo VG, McDonald LC, et al: Clinical practice guidelines for Clostridium difficile infection in adults: 2010 update by the society for healthcare epidemiology of America (SHEA) and the infectious diseases society of America (IDSA). Infect Control Hosp Epidemiol 2010, 31(5):431-455.

4. Ricciardi R, Rothenberger DA, Madoff RD, Baxter NN: Increasing prevalence and severity of Clostridium difficile colitis in hospitalized patients in the United States. Arch Surg 2007, 142(7):624-631. discussion 31.

5. Labbe AC, Poirier L, Maccannell D, Louie T, Savoie M, Beliveau C, et al: Clostridium difficile infections in a Canadian tertiary care hospital before and during a regional epidemic associated with the BI/NAP1/027 strain. Antimicrob Agents Chemother 2008, 52(9):3180-3187.

6. Wysowski DK: Increase in deaths related to enterocolitis due to Clostridium difficile in the United States, 1999-2002. Public Health Rep 2006, 121(4):361-362.

7. Miller M, Gravel D, Mulvey M, Taylor G, Boyd D, Simor A, et al: Health careassociated Clostridium difficile infection in Canada: patient age and infecting strain type are highly predictive of severe outcome and mortality. Clin Infect Dis 2010, 50(2):194-201.

8. Kim J, Smathers SA, Prasad P, Leckerman KH, Coffin S, Zaoutis T: Epidemiological features of Clostridium difficile-associated disease among inpatients at children's hospitals in the United States, 2001-2006. Pediatrics 2008, 122(6):1266-1270.

9. Deshpande A, Pant C, Anderson MP, Donskey CJ, Sferra TJ: Clostridium difficile infection in the hospitalized pediatric population: increasing trend in disease incidence. Pediatr Infect Dis J 2013, 32(10):1138-1140.

10. Nylund CM, Goudie A, Garza JM, Fairbrother G, Cohen MB: Clostridium difficile infection in hospitalized children in the United States. Arch Pediatr Adolesc Med 2011, 165(5):451-457.

11. Benson L, Song X, Campos J, Singh N: Changing epidemiology of Clostridium difficile-associated disease in children. Infect Control Hosp Epidemiol 2007, 28(11):1233-1235.

12. Khanna S, Baddour LM, Huskins WC, Kammer PP, Faubion WA, Zinsmeister $A R$, et al: The epidemiology of Clostridium difficile infection in children: a population-based study. Clin Infect Dis 2013, 56(10):1401-1406.
13. Sammons JS, Toltzis $P$, Zaoutis TE: Clostridium difficile infection in children. JAMA Pediatr 2013, 167(6):567-573.

14. Gravel D, (CNISP) CNISP: Clostridium difficile Associated Diarrhea in Acute-Care Hospitals Participating in CNISP: November 1, 2004 to April 30, 2005 Final Report. http://www.phac-aspc.gc.ca/nois-sinp/pubs-eng.php Sept. 5 2007; Accessed July 92013.

15. Kim J, Shaklee JF, Smathers S, Prasad P, Asti L, Zoltanski J, et al: Risk factors and outcomes associated with severe clostridium difficile infection in children. Pediatr Infect Dis J 2012, 31(2):134-138.

16. Tang P, Roscoe M, Richardson SE: Limited clinical utility of Clostridium difficile toxin testing in infants in a pediatric hospital. Diagn Microbiol Infect Dis 2005, 52(2):91-94.

17. Mulvey MR, Boyd DA, Gravel D, Hutchinson J, Kelly S, McGeer A, et al: Hypervirulent Clostridium difficile strains in hospitalized patients, Canada. Emerg Infect Dis 2010, 16(4):678-681.

18. Mulvey M, Boyd D, Du T, Hizon R, Miller M, Gravel D, et al: The molecular epidemiology of Clostridium difficile in Canadian hospitals: 2007-2011. Can J Infect Dis Med Microbiol 2013, 24(Suppl B):5B.

19. Zilberberg MD, Tillotson GS, McDonald C: Clostridium difficile infections among hospitalized children, United States, 1997-2006. Emerg Infect Dis 2010, 16(4):604-609.

20. Lee J, Tashjian DB, Moriarty KP: Is partial colectomy the operation of choice in pediatric Clostridium difficile colitis? Pediatr Surg Int 2012, 28(6):603-607.

21. Ananthakrishnan AN, McGinley EL, Binion DG: Excess hospitalisation burden associated with Clostridium difficile in patients with inflammatory bowel disease. Gut 2008, 57(2):205-210.

22. Lamouse-Smith ES, Weber S, Rossi RF, Neinstedt LJ, Mosammaparast N, Sandora TJ, et al: Polymerase chain reaction test for clostridium difficile toxin $B$ gene reveals similar prevalence rates in children with and without IBD. J Pediatr Gastroenterol Nutr 2013, 57(3):293-297.

23. Morgan OW, Rodrigues B, Elston T, Verlander NQ, Brown DFJ, Brazier J, et al: Clinical severity of Clostridium difficile PCR ribotype 027: a case-case study. PLoS One [Electronic Resource] 2008, 3(3):e1812.

24. Gravel D, Miller M, Simor A, Taylor G, Gardam M, McGeer A, et al: Health care-associated Clostridium difficile infection in adults admitted to acute care hospitals in Canada: a Canadian Nosocomial Infection Surveillance Program Study. Clin Infect Dis 2009, 48(5):568-576.

25. Loo VG, Bourgault A-M, Poirier L, Lamothe F, Michaud S, Turgeon N, et al: Host and pathogen factors for Clostridium difficile infection and colonization. N Engl J Med 2011, 365(18):1693-1703.

26. Toltzis P, Kim J, Dul M, Zoltanski J, Smathers S, Zaoutis T: Presence of the epidemic North American pulsed field type 1 Clostridium difficile strain in hospitalized children. J Pediatr 2009, 154(4):607-608.

27. Stoesser N, Crook DW, Fung R, Griffiths D, Harding RM, Kachrimanidou M, et al: Molecular epidemiology of Clostridium difficile strains in children compared with that of strains circulating in adults with Clostridium difficileassociated infection. J Clin Microbio/ 2011, 49(11):3994-3996

28. Pai S, Aliyu SH, Enoch DA, Karas JA: Five years experience of Clostridium difficile infection in children at a UK tertiary hospital: proposed criteria for diagnosis and management. PLoS One 2012, 7(12):e51728.

\section{doi:10.1186/1471-2431-14-28}

Cite this article as: Schwartz et al: Severe clinical outcome is uncommon in Clostridium difficile infection in children: a retrospective cohort study. BMC Pediatrics 2014 14:28. 